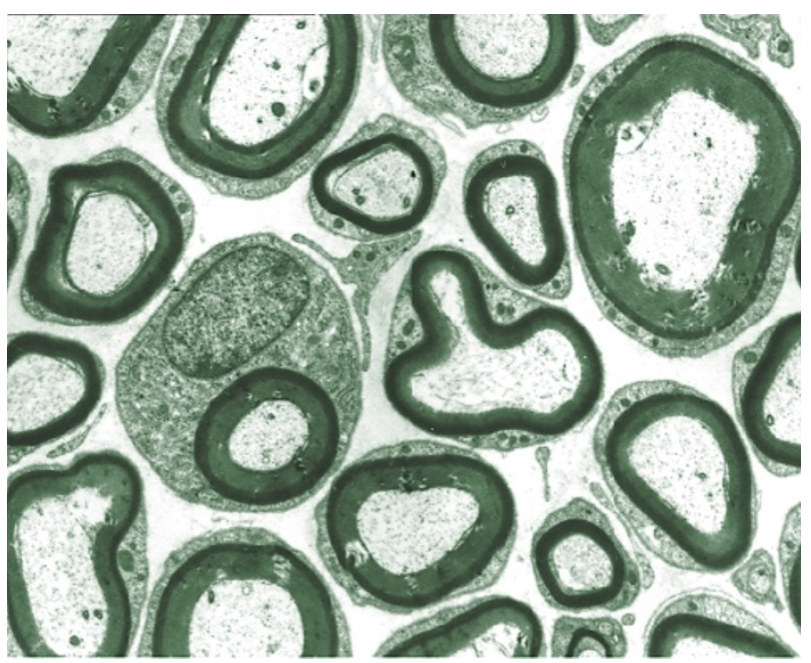

Electron micrograph of sciatic nerve. Courtesy of J. Milbrandt, Washington University, St Louis, Missouri, USA.

\section{GLIA}

\section{Profile of a neuropathy}

Many demyelinating diseases have a genetic basis. Although some of these disorders are associated with mutations in myelin proteins such as myelin protein zero and periaxin, this is not always the case. For example, mutations in the transcription factor $E G R 2$ are found in some patients with inherited peripheral neuropathies. Intriguingly, these neuropathies are transmitted in a dominant manner, but mice heterozygous for an Egr2 null allele are normal. This observation prompted the suggestion that the mutant EGR2 gene encodes a dominant-negative mutant protein in people with the disease. But as Nagarajan et al. report in Neuron, testing this seemingly straightforward hypothesis has led to deeper, unanticipated insights into the mechanisms behind these demyelinating diseases.

The authors first used a conventional approach to test whether mutant Egr2 acted as a dominant-negative inhibitor and could block the expression of a reporter gene. As the results were negative, they wondered whether any dominant-negative activity of the mutant transcription factor would only become manifest if the target genes were in their endogenous loci. However, to explore this idea required the previous identification of Egr2 targets. So, the authors used microarray expression profiling of Schwann cells to identify genes induced by Egr 2 expression, and found that several myelin genes were under the control of this transcription factor. Now, when they tested the effect of mutant Egr2 on the expression of these myelin genes in Schwann cells, Nagarajan et al. observed the dominant-negative activity that they had originally tried to find.

So, an inability of the Schwann cells to produce crucial myelin proteins and execute the myelination programme seems to be at the core of the inherited peripheral neuropathies. Importantly, Nagarajan et al. obtained evidence that reintroducing the normal Egr2 gene to cells that express low levels of the protein can lead to the expression of the missing myelin proteins. This finding raises the possibility that the myelination defect might be reversible and opens new avenues for the development of therapeutic measures to treat these debilitating disorders.

Juan Carlos López

(2) References and links

ORIGINAL RESEARCH PAPER Nagarajan, R. et al. EGR2 mutations in inherited

neuropathies dominant-negatively inhibit myelin gene expression. Neuron $\mathbf{3 0}, 355-368$ (2001)

FURTHER READING Warner, L. E. et al. Mutations in the early growth response 2 (EGR2) gene are associated with hereditary myelinopathies. Nature Genet. 18, 382-384 (1998)

\title{
Worth the wait
}

How do we control our impulses? If the choice is between a small treat now and a large treat later, most of us would choose the large treat, even though we have to wait for it. The inability to overcome the desire for immediate reward in this way is known as impulsive choice; it has been implicated in addiction, attention deficit hyperactivity disorder (ADHD) and some personality disorders, but its cause is unclear.

Rudolf Cardinal and colleagues have investigated the neural substrates of impulsive choice by looking at the preferences of rats for a small, immediate reward or a larger, delayed one. They found that lesions of the nucleus accumbens core reduced the rats' preference for the larger, delayed reward, apparently by making them unable to overcome their impulsive desire for immediate gratification. Unlesioned rats would choose the larger reward on some trials even if it was delayed by up to 60 seconds, but lesioned animals usually chose the smaller reward even if the delay to the larger one was only 10 seconds.

Cardinal et al. also tested rats in which either the medial prefrontal cortex (mPFC) or the anterior cingulate cortex (ACC) was lesioned. Both of these areas project to the core of the nucleus accumbens and have been implicated in impulsive behaviour. Surprisingly, neither of these lesions induced impulsive choice in

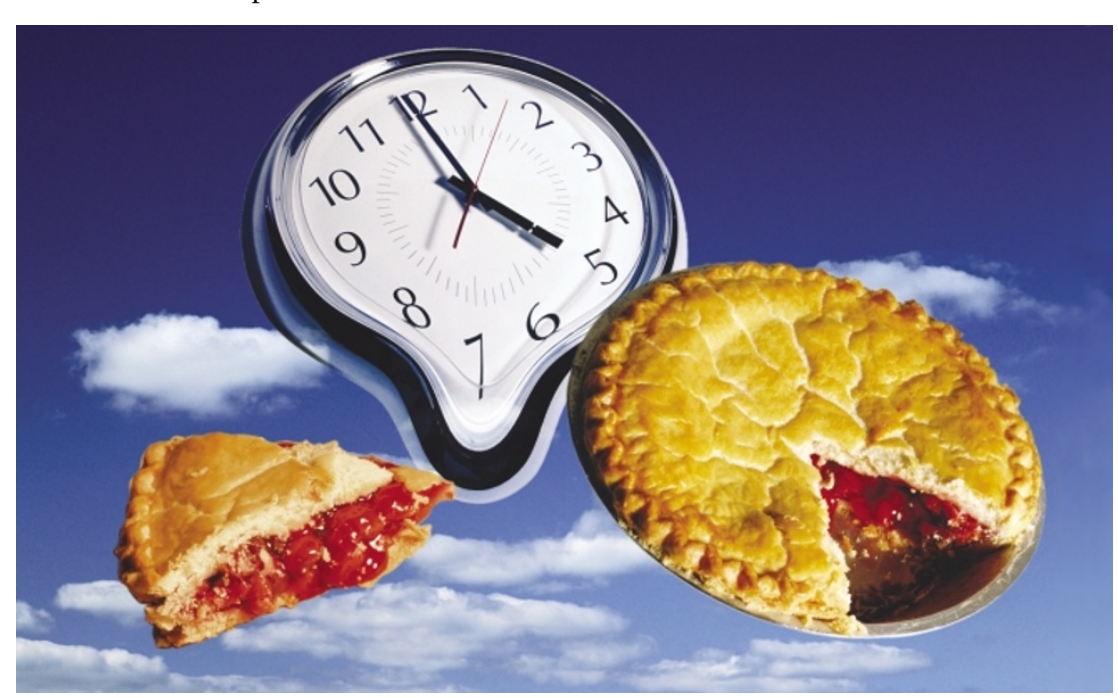

the rats. ACC lesions had no effect on the task, even though such lesions have been implicated in response disinhibition. The authors attribute this apparent contradiction to a dissociation between motor impulsivity and impulsive choice. Rats with mPFC lesions chose the large, delayed reward less frequently than shams at zero delay, but more frequently at the maximum delay. This may reflect an interaction of the mPFC lesion with the specific task used.

It remains to be seen which accumbens afferents are involved in controlling impulsive choice. But these data suggest that the nucleus accumbens may be important in our ability to make rational decisions when faced with this kind of choice, and in its disruption in various disorders. Cardinal et al. also suggest that the results may indicate that the nucleus accumbens is the site of action of methylphenidate (Ritalin), which modifies dopamine signalling and can relieve the symptoms of ADHD.

Rachel Jones

(D) References and links ORIGINAL RESEARCH PAPER Cardinal, R. N. et al. Impulsive choice induced in rats by lesions of the nucleus accumbens core. Science 24 May 2001 (10.1126/science.1060818)

FURTHER READING Evenden, J. L. Varieties of impulsivity. Psychopharmacology 146, 348-361 (1999) | Sagvolden, T. \& Sergeant, J. A. Attention deficit/hyperactivity disorder - from brain dysfunctions to behaviour. Behav. Brain Res. 94 ,

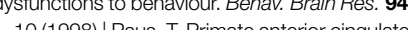
cortex: where motor control, drive and cognition interface. Nature Rev. Neurosci. 2, 417-424 (2001) 